\title{
Contribution to Better Handling of Irregular Problems in HPF2
}

\author{
Thomas Brandes ${ }^{1}$, Frédéric Brégier ${ }^{2}$, Marie Christine Counilh ${ }^{2}$, Jean Roman ${ }^{2}$ \\ 1 GMD/SCAI, Institute for Algorithms and Scientific Computing, \\ German National Research Center for Computer Science, Schloss Birlinghoven, \\ PO Box 1319, 53754 St. Augustin, Germany \\ 2 LaBRI, ENSERB and Université Bordeaux I, 33405 Talence Cedex, France
}

\begin{abstract}
In this paper, we present our contribution for handling irregular applications with HPF2. We propose a programming style of irregular applications close to the regular case, so that both compiletime and run-time techniques can be more easily performed. We use the well-known tree data structure to represent irregular data structures with hierarchical access, such as sparse matrices. This algorithmic representation avoids the indirections coming from the standard irregular programming style. We use derived data types of Fortran 90 to define trees and some approved extensions of HPF2 for their mapping. We also propose a run-time support for irregular applications with loop-carried dependencies that cannot be determined at compile-time. Then, we present the TriDenT library, which supports distributed trees and provides runtime optimizations based on the inspector/executor paradigm. Finally, we validate our contribution with experimental results on IBM SP2 for a sparse Cholesky factorization algorithm.
\end{abstract}

\section{Introduction}

High Performance Fortran (HPF) $[10]$ is the current standard language for writing data parallel programs for shared and distributed memory parallel architectures. HPF is well suited for regular applications; however, many scientific and engineering applications (fluid dynamics, structural mechanics, ... ) use irregular data structures such as sparse matrices. Irregular data need a special representation in order to save storage requirements and computation time, and the data accesses use indirect addressing through pointers stored in index arrays (see for example SPARSKIT [14]). In these applications, patterns of computations are generally irregular and special data distributions are required to both provide high data locality for each processor and good load balancing. Then, compiletime analysis is not sufficient to determine data dependencies, location of data and communication patterns; indeed, they are only known at run-time because they depend on the input data. Therefore, it is currently difficult to achieve good performance with these irregular applications.

The second version of the language, HPF2 [10], introduces new features for efficient irregular data distributions and for data locality specification in irregular computations. To deal with the lack of compile-time information on irregular 
codes, run-time compilation techniques based on the inspector/executor method have been proposed and widely used.

Major works include PARTI [16] and CHAOS [12] libraries used in Vienna Fortran and Fortran90D [13] compilers, and PILAR library [11] used in PARADIGM compiler [2]. They are efficient for solving iterative irregular problems in which communication and computation phases alternate. RAPID [9] is another runtime system based on a computation specification library for specifying irregular data objects and tasks manipulating them. It is not limited to iterative computations and can address algorithms with loop-carried dependencies.

In order to reduce the overhead due to these run-time techniques, the technique called sparse-array rolling (SAR) [15] exploits compile-time information about the representation of distributed sparse matrices for optimizing data accesses. In the Vienna Fortran Compilation System [6], a new directive (SPARSE directive) [15] specifies the sparse matrix structure used in the program to improve the performance of existing sparse codes.

Bik and Wijshoff [3] propose another approach based on a "sparse compiler" that converts a code operating on dense matrices and annoted with sparsity related information into an equivalent sparse code.

Our approach consists of combining compile-time analysis and run-time techniques, but in a way that differs from the previously cited works. We propose a programming style of irregular applications close to the regular case so that both compile-time and run-time techniques can be more easily performed. In order to do so, we use the well-known tree data structure to represent sparse matrices and, more generally, irregular data structures with a hierarchical access. This algorithmic representation masks the implementation structure and avoids the indirections coming from the standard irregular programming style. Like in the SAR approach [15], the compiler knows more information about the distributed data, but unlike this approach, information naturally result from the representation of the irregular data structure itself. Our approach is not restricted to sparse matrices but it necessitates a coding of irregular applications using our tree structure. In order to increase portability, we use Derived Data Types (DDT) of Fortran 90 to define trees and hence irregular data structures, and we use some approved extensions of HPF 2 for their mapping. In all the following of this paper, we will refer to our language proposition as HPF2/Tree. We also propose a run-time support for irregular applications with loop-carried dependencies that cannot be determined at compile-time. Each iteration is performed on a subset of processors only known at run-time. This support is based on an inspector/executor scheme which builds the processor sets, the loop indices for each processor and generates the necessary communications. So, the first step of our work has been to develop a library, called TriDenT, which supports distributed trees and implements the above-mentioned optimizations. The performance of this library have been demonstrated on irregular applications such as sparse Cholesky factorization. The following step of this work is to provide an HPF2/Tree compiler; we are currently working on the integration of the TriDenT library in the ADAPTOR compilation platform [5]. 
This paper (see [4] for a more detailed version) is organized as follows. In Section 2, we give a short background on a sparse column Cholesky factorization algorithm which will be our illustrative and experimental example for the validation of our approach. Section 3 presents HPF2/Tree and section 4 describes the TriDenT library. Section 5 gives an experimental study on IBM SP2 for sparse Cholesky factorization applied on real size problems, and provides an analysis of the performance. Finally, section 6 gives some perspectives of this work.

\section{An Illustrative Example}

The Cholesky factorization of sparse symmetric positive definite matrices is an extremely important computation arising in many scientific and engineering applications. However, this factorization step is quite time-consuming and is frequently the computational bottleneck in these applications. Consequently, it is a significant interesting example for our study. The goal is to factor a sparse symmetric positive definite $n \times n$ matrix $A$ into the form $A=L L^{T}$, with $L$ lower triangular. Two steps are typically performed for this computation.

First, we perform a symbolic factorization to compute the non-zero structure of $L$ from the ordering of the unknowns in $A$; this ordering, for example using a nested dissection strategy must reduce the fill in and increase the parallelism in the computations. This (irregular) data structure is allocated and its initial non-zero coefficients are those of $A$. In the pseudo-code given below, this step is implicitly contained in the instruction $L=A$. From this symbolic factorization, one can deduce for each $k$ the two following sets defined as the sparsity structure of row $k$ and the sparsity structure of column $k$ of $L$ :

$$
\begin{aligned}
& \operatorname{Struct}\left(L_{k *}\right)=\left\{j<k \text { such that } l_{k j} \neq 0\right\} \\
& \operatorname{Struct}\left(L_{* k}\right)=\left\{i>k \text { such that } l_{i k} \neq 0\right\} .
\end{aligned}
$$

Second, the numerical factorization computes the non-zero coefficients of $L$ in the data structure. This step, which is the most time-consuming, can be performed by the following sparse column-Cholesky factorization algorithm (see for example $[7,1]$ and included references).

$$
\begin{aligned}
& \text { 1. } L=\mathrm{A} \\
& \text { 2. for } \mathrm{k}=1 \text { to } \mathrm{n} \text { do } \\
& \text { 3. for } j \in \operatorname{Struct}\left(L_{k_{*}}\right) \text { do } \\
& \text { 4. } \quad \text { for } i \in \operatorname{Struct}\left(L_{* j}\right), i \geq k \operatorname{do} \% \operatorname{cmod}(\mathrm{k}, j, \mathrm{i}) \% \\
& \text { 5. } l_{i k}=l_{i k}-l_{k j} * l_{i j} \\
& \text { 6. } l_{k k}=\sqrt{l_{k k}} \% \operatorname{cdiv}(\mathrm{k}) \% \\
& \text { 7. for } i \in \operatorname{Struct}\left(L_{* k}\right) \text { do } \\
& \text { 8. } \quad l_{i k}=l_{i k} / l_{k k}
\end{aligned}
$$

where $\operatorname{cmod}(k, j, i)$ represents the modification of the rows of the column $k$ by the corresponding terms in column $j$, and $c d i v(k)$ represents the division of the column $k$ by the scalar $\sqrt{l_{k k}}$.

This algorithm is said to be a left-looking algorithm, since at each stage it accesses needed columns to the left of the current column in the matrix. It is also referred to as a fan-in algorithm, since the basic operation is to combine the effects of multiple previous columns on a single subsequent column. 
If we consider now the parallelism induced by sparsity and achieved by distributing the columns on processors (so, we exploit the parallelism of the outer loop of instruction 2.), we can see that a given column $k$ depends only of columns belonging to $\operatorname{Struct}\left(L_{k *}\right)$; so, we have loop-carried dependencies in this algorithm. In order to take advantage of this structural parallelism, we use an irregular distribution called subtree-to-subcube mapping and which leads to an efficient reduction of communication while keeping a good load balance between processors; this mapping is computed algorithmically from the sparse data structure for $L$ (see for example [7] and included references).

\section{HPF2/Tree}

\subsection{Representation of Irregular Data Structures with Trees}

In this paper, we consider irregular data structures with a hierarchical access and we propose the use of a tree data structure for their representation. For example, the sparse matrix used in the previous oriented column Cholesky algorithm must be a data structure with a hierarchical column access and the user can represent it by a tree with three levels numbered from 0 to 2 . The $k$-th node on level 1 represents the $k$-th column. Its $p$-th son represents the $p$-th nonzero element defined by its value and row number. Fig. 1 gives a sparse matrix and its corresponding tree. In order to distinguish the levels, we number the columns with roman numbers and the rows with arabic numbers.

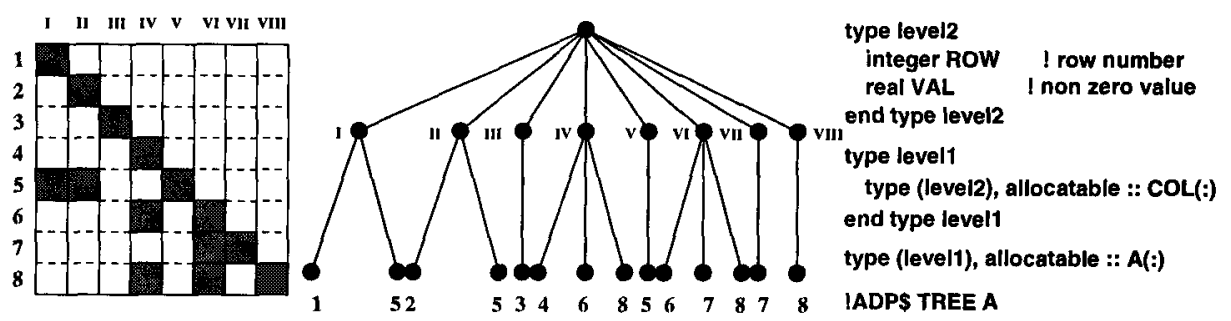

Fig. 1. A Sparse Matrix and its HPF2/Tree Declaration.

\subsection{Tree Representation and TREE Directive in HPF2}

Trees are embedded in the HPF2 programming language by using the Derived Data Type (DDT) of Fortran 90. For every level of a tree (except level 0), a DDT must be defined. It contains all the data type definitions for this level and (except for the level with the greatest number) the declaration of an array variable whose type is the one associated with the following level. A tree is then declared as an array of the type the DDT associated with the level 1 (cf. Fig. 1).

The TREE directive distinguishes tree variables from other variables using DDT because these variables are used in a restricted way; in particular, pointer 
attributes of Fortran 90 and recursions are not allowed. So, our tree data structures must not be recursively defined and cannot be used for example to represent quadtree decompositions of matrices [8]. However, this limitation leads to an efficient implementation of trees by the compiler (cf. 4.1).

The accesses to a specific tree element or to a subtree are performed according to the usual Fortran 90 notation. The advantage of this programming style is the analogy between the level notion for trees and the classical dimension notion for arrays used in regular applications. Then, the compiler can perform the classical optimizations based on dependence analysis much more easily than with indirection arrays currently used in irregular applications.

\subsection{Distribution of Trees}

In order to support irregular applications, HPF2/Tree includes the GEN_BLOCK and INDIRECT distribution formats and allows the mapping of the components of DDT according to HPF2.0 approved extensions [10]. The constraints for the mapping of derived type components allow the mapping of structure variables at only one level, the reference level. For the two distributions of tree A given at Fig. 2, the reference level is level 1.

In HPF2/Tree, the levels preceding the reference level are replicated while the levels following it are distributed according to the distribution of this reference level. This distribution implies an implicit alignment of data with the data of the reference level. This data locality is an important advantage of tree distribution and a compiler can exploit it to generate an efficient code. This is clearly more difficult to obtain when a sparse storage format, such as CSC format [14], is used because the indirection and data arrays are of different sizes and their distribution formats also differ.
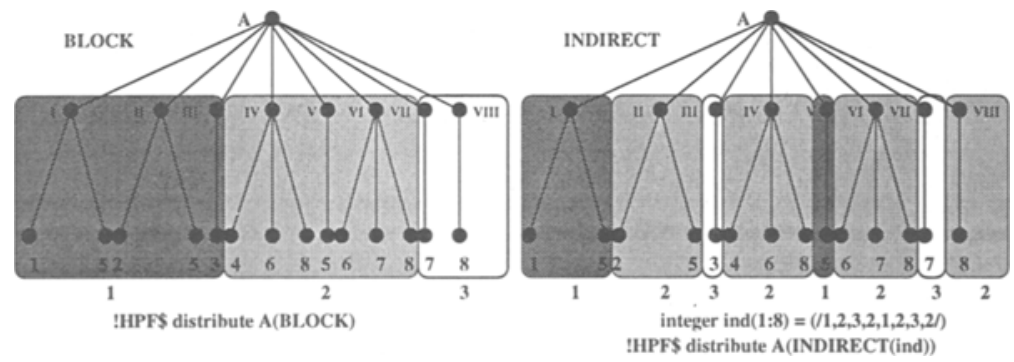

Fig. 2. Tree Distributions with 3 Processors.

\subsection{Application to the Sparse Fan-In Cholesky Algorithm}

This section describes an HPF2/Tree code (cf. Program 3 (a)) for the sparse fanin Cholesky algorithm presented in section 2. This code uses the tree A declared in Fig. 1 and another tree, named $B$, used to store the dependencies between columns. Then, the first value of $B(K) \% \operatorname{COL}(:) \% D E P$ is $K$, and the other ones are the column numbers given by $\operatorname{Struct}\left(L_{k *}\right)$. In section 4.3 , we will show that these 
data can be computed during an appropriate inspection phase so that this tree $\mathrm{B}$ can be avoided. The distribution of tree $A$ uses a specific INDIRECT distribution of nodes on level 1 according to a subtree-to-subcube mapping (cf. section 2). Then, the tree $B$ is aligned with the tree A using the HPF ALIGN directive.

In the outer $\mathrm{K}$ loop, the $\mathrm{ON}$ directive asserts that the statements at iteration $K$ are performed by the set of processors that own at least one column with a non-zero value in row $\mathrm{K}$. The internal $\mathrm{L}$ loop performs a reduction inside this set of processors using the NEW variable TMP_VAL specified in the ON directive. Each iteration $\mathrm{L}$ is performed by the processor which owns the column with number $\mathrm{J}=\mathrm{B}(\mathrm{K}) \% \operatorname{COL}(\mathrm{L}) \% \mathrm{DEP}$; so, the computation of the contributions for the column $\mathrm{K}$ is distributed. Moreover, a compiler might identify that this reduction uses an all-to-one scheme due to the instruction following the loop. The tree notation which avoids indirection in the code, and the data locality and alignment provided by the distribution of the tree make the compiler able to extract the needed communications outside this internal loop (for $A(K) \% \operatorname{COL}(:) \% R O W$ and $\mathrm{B}(\mathrm{K}) \% \operatorname{COL}(:) \%$ DEP variables). This would not be possible by using a sparse storage format (such as $\mathrm{CSC}$ ) due to the indirections.
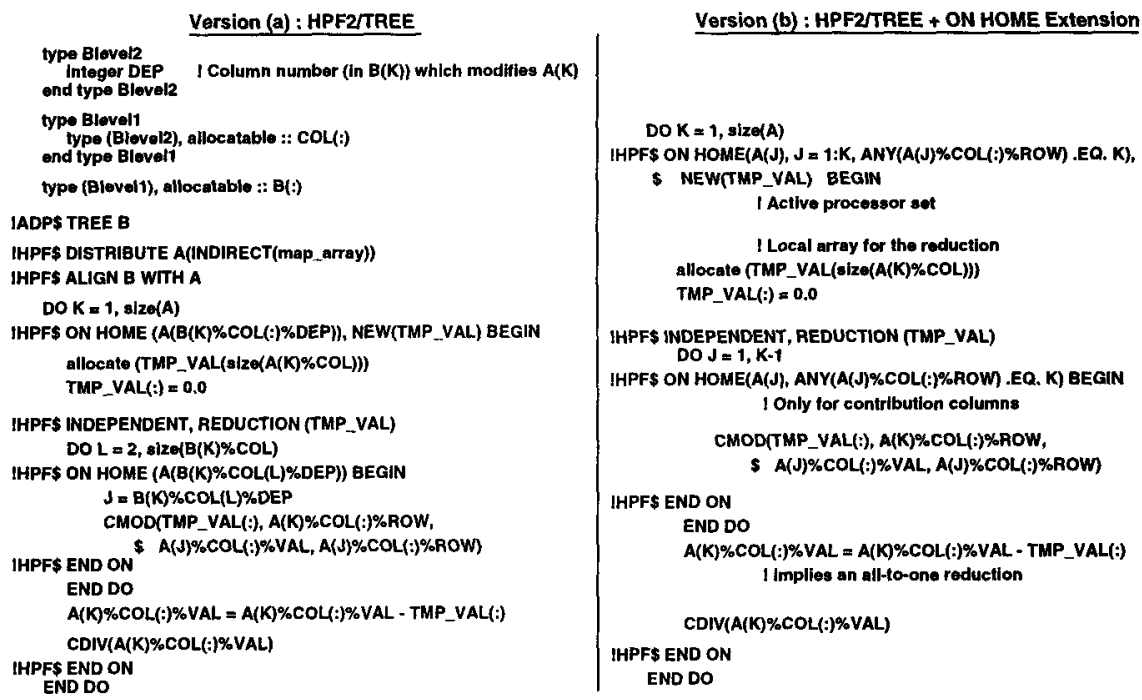

Program 3. 2 Versions of the Fan-In Cholesky Algorithm.

However, this example also shows that run-time techniques are required because the set of active processors specified by the on directive cannot be known until run-time. In the following section, we describe the TriDenT run-time system which provides supports for tree and for such irregular active processor sets.

\section{The TriDenT Library}

TriDen $\mathrm{T}$ is a library which consists of two parts: a set of routines for the manipulation of trees, and another one for the optimization of computations and communications in irregular processor sets based on the inspector/executor paradigm. 
We currently use this library to validate the tree approach and our optimization proposals by writing HPF 2 codes with explicit calls to TriDenT primitives. Furthermore, the TriDenT library has been designed to be integrated as easily as possible in the compilation platform ADAPTOR [5] in order to automatically translate HPF2/Tree codes in SPMD codes with calls to TriDenT primitives. ADAPTOR also supports HPF2 features and is based on the DALIB (Distributed Array LIBrary) run-time system for distributed arrays. The following sections present the two components of the TriDenT library.

\subsection{Support for Distributed Trees}

TriDenT implements trees by using an array representation. The TREE directive allows the compiler to generate one-dimensional arrays for the implementation of the Derived Data Types associated with the tree; this implementation consists of one array for each scalar variable of a DDT and of some pointer arrays (cf. Fig. 4). Hence, TriDen'T provides efficient access functions to tree elements and to subtrees; moreover, the array implementation allows to take advantage of the potential cache effects.

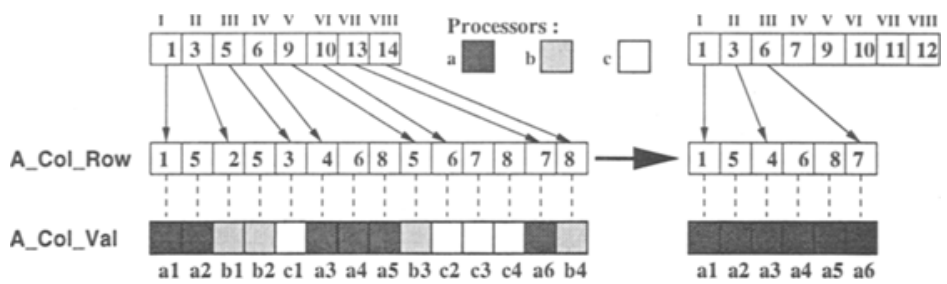

Fig. 4. Indirect Distribution and Local Data for Processor a.

The distribution of trees (cf. 3.3) is performed by using the technique described in [13]. It consists in distributing all the data arrays, for the levels from the reference level, with GEN BLOCK distribution format according to the tree distribution format. Then, the processor local data are stored in a contiguous memory space (cf. Fig. 4 for the indirect distribution of Fig. 2).

\subsection{Support for Irregular Processor Subsets}

TriDenT is a run-time system which integrates the active processor set notion introduced in HPF2 by the ON directive. This directive specifies how computation is partitioned among processors and therefore may affect the efficiency of computation. Then TriDenT includes context push and pop as well as reduction and broadcast for active processor sets. Moreover, if the ON directive includes an indirection (e.g. ON HOME (A(INDIR(1:4)))), the set can be only determined at run-time and so an inspection phase is necessary. TriDenT provides further optimizations for handling ON directive used inside a global DO loop with loopcarried dependencies which can be determined before this loop.

Our main goal is to improve the efficiency of the global computation (inspection and execution), even if the executor is used only once. The inspector consists of three parts. 
The Set Inspector analyzes the ON directive of the global loop in order to create in advance the processor subsets associated with each iteration. This will avoid during the execution of the global loop the synchronizations due to the set building messages. In order to allow an automatic generation of these irregular sets, we propose to extend the on directive by specifying the algorithmic properties to be verified by the variables in the HOME clause (cf. Program 3 (b)). The Loop Index Inspector determines the useful loop indices for each processor. The Communication Inspector inspects two kinds of communications: those to extract out of the global loop and across-iterations communications. Moreover, the executor optimizes the communications by splitting the send and receive operations and integrating them in the code so as to minimize the synchronisations.

\subsection{Application to the Sparse Fan-In Cholesky Algorithm}

We briefly described the optimizations achieved by inpection mechanisms on the HPF2/Tree code for the sparse fan-in Cholesky algorithm given in Program 3 (b). This code uses the HOME clause extension introduced in section 4.2 and avoids the effective storage of the dependence tree B used in Program 3 (a) (whose size is of the same order of magnitude as $A(:) \% \operatorname{COL}(:) \% R O W)$.

The Set Inspector involved by the extended on directive for the global loop directly generates from the $A(:) \% \operatorname{COL}(:) \%$ ROW data (and so without the tree $B$ ) the processor set for each iteration $K$ (ACTIVE_PROC $(K))$.

In this example, the Loop Index Inspection for the inner loop allows to avoid the scan of the A(J)\%COL(:)\%ROW, and to directly use the valid iteration index sets for each processor.

The compiler can identify A(K)\%COL ( : )\%ROW as loop invariant, and can detect that these data must be sent to the ACTIVE_PROC(K) set; then the Communication Inspector can extract the emission outside the global loop and keep the reception inside iterations.

\section{$5 \quad$ Experimental Results}

We validate our contribution with experimental results for a sparse fan-in Cholesky factorization on IBM SP2 with 16 processors. The test matrix is a $n \times n$ sparse matrix with $n=65024$ achieved from a $2 \mathrm{D}$-grid finite element problem. This matrix is distributed with an INDIRECT mapping according to the subtreeto-subcube distribution (cf. section 2). We compare the three versions described at Fig. 5. The $\mathbf{A}$ version uses the tree B (cf. Program 3 (a)), whereas the $\mathbf{B}$ and $\mathbf{C}$ versions use the proposed extended HOME clause (cf. Program 3 (b)). In all the following, the global time consists in the summation of inspection and execution times. All these versions use the Processor Set Inspection. We have verified the effectiveness of this basic inspection by comparing the execution of $\mathbf{A}$ with another version which doesn't use the processor subsets (35 seconds against 460 seconds for the global time with 16 processors). 
\begin{tabular}{|c|c|c|c|}
\hline Name & Irregular Set Insp. & Loop Index Insp. Communication Insp. Dep. (T)ree B or (0)N HoME Ext.
\end{tabular}

\begin{tabular}{|c|c|c|c|c|}
\hline $\mathbf{A}$ & Yes & No & No & T \\
\hline $\mathbf{B}$ & Yes & Yes & No & O \\
\hline $\mathbf{C}$ & Yes & Yes & Yes & O \\
\hline
\end{tabular}

Fig. 5. Characteristics of the Three Versions of the Fan-In Cholesky Factorization

The Fig. 6 presents the relative efficiency with regard to version $\mathbf{A}$, first for the execution time only, and second for the global time. The first graph demonstrates the improvment obtained : from 2 to $8 \%$ for the Loop Index Inspection (B), from 3 to $13 \%$ with Communication Inspection added (C). The second graph illustrates that the global time, even for only one executor phase, can be better than for the reference version $\mathbf{A}$. It appears that the Loop Index Inspection leads to an interesting improvment ( $4 \%$ for $\mathbf{B}$ ). However, the cost of the Communication Inspection is just recover by the executor on 16 processors; nevertheless, one can expect a greater improvement with more processors.

In all our experimentations, we can notice that inspection times are proportional to global time. This property is important for the scalability of our execution support. For example, this time represents between $9 \%$ and $15 \%$ for version $\mathbf{C}$ according to the number of processors.
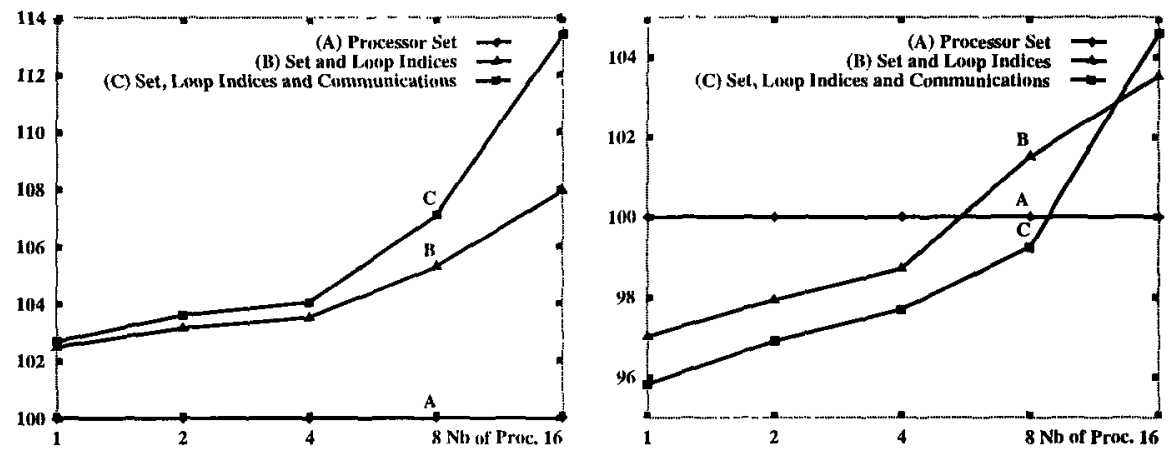

Fig. 6. Relative Efficiencies (Execution Time and Global Time).

So, these experimental results validate our run-time system. Of course, if the factorization step is executed many times, then the inspector cost will be negligible compared with the gain achieved by multiple executions. See [4] for other measures which validate this approach.

\section{Conclusion and Perspectives}

In this paper, we present a contribution to better handling irregular problems in a HPF2 context. We use a tree representation to take data or distribution irregularity into account. This can help the compiler to perform standard analysis and optimizations. We also introduce an extension for the ON HOME directive in order to enable automatic irregular processor set creations, and we propose a run-time support based on inspection/execution paradigm for active processor sets, loop indices and communications for algorithms with loop-carried dependencies. Our approach is validated by experimental results for sparse Cholesky factorization. 
Our future work is to extend the properties of our trees (geometrical distributions as BRS or MRD [15], flexible trees in order to enable reallocations and redistributions), and to improve the performance of our Inspector/Executor system (use of the task parallelism induced by the loops). Finally, we are currently working on the integration of TriDen $T$ in the compilation platform ADAPTOR.

\section{References}

1. C. Ashcraft, S. C. Eisenstat, J. W.-H. Liu, and A. H. Sherman. A Comparison of Three Column Based Distributed Sparse Factorization Schemes. In Fifth SIAM Conference on Parallel Processing for Scientific Computing, 1991.

2. P. Banerjee, J. A. Chandy, M. Gupta, J. G. Holm, A. Lain, D. J. Palermo, S. Ramaswamy, and E. Su. The PARADIGM Compiler for Distributed-Memory Message Passing Multicomputers. In the First International Workshop on Parallel Processing, Bangalore, India, December 1994.

3. A. J.C. Bik and H. A.G. Wijshoff. Simple Quantitative Experiments with a Sparse Compiler. In A. Ferreira J. Rolim Y. Saad and T. Yang editors, editors, Proc. of Third International Workshop, IRREGULAR'96, volume 1117 of Lecture Notes in Computer Science, pages 249-262, Springer, August 1996.

4. T. Brandes, F. Brégier, M.C. Counilh, and J. Roman. Contribution to Better Handling of Irregular Problems in HPF2. Technical Report RR 120598, LaBRI, May 1998.

1. T. Brandes and F. Zimmermann. Programming Environments for Massively Parallel Distributed Systems, chapter ADAPTOR - A Transformation Tool for HPF Programs, pages 91-96. In K.M. Decker and R.M. Rehmann, editors, Birkhauser Verlag, April 1994.

6. B. Chapman, S. Benkner, R. Blasko, P. Brezany, M. Egg, T. Fahringer, H.M. Gerndt, J. Hulman, B. Knaus, P. Kutschera, H. Moritsch, A. Schwald, V. Sipkova, and H. Zima. Vienna Fortran Compilation System, User's Guide Edition, 1993.

7. K. A. Gallivan et al. Parallel Algorithms for Matrix Computations. SIAM, Philadelphia, 1990.

8. J. D. Frens and D. S. Wise. Auto-blocking Matrix-Multiplication or Tracking BLAS3 Performance with Source Code. Technical Report 449, Computer Scince Department, Indiana University, December 1996.

9. C. Fu and T. Yang. Run-Time Techniques for Exploiting Irregular Task Parallelism on Distributed Memory Architectures. Journal of Parallel and Distributed Computing, 42:143-156, 1997.

10. HPF Forum. High Performance Fortran Language Specification, January 1997. Version 2.0.

11. A. Lain. Compiler and Run-time Support for Irregular Computations. PhD thesis, Illinois, 1996.

12. S.S. Mukherjee, S. D. Sharma, M. D. Hill, J. R. Larus, A. Rogers, and J. Saltz. Efficient Support for Irregular Applications on Distributed-Memory Machines. In ACM SIGPLAN Symposium on Principles 63 Practice of Parallel Programming (PPoPP), July 1995.

13. R. Ponnusamy, Y.S. Hwang, R. Das, J. Saltz, A. Choudhary, and G. Fox. Supporting Irregular Distributions in Fortran 90D/HPF Compilers. IEEE Parallel and Distributed Technology, 1995. Technical Report CS-TR-3268 and UMIACS-TR-9457. 
14. Y. Saad. SPARSKIT : a Basic Tool Kit for Sparse Matrix Computations - Version 2. Technical report, CSRD, University of Illinois, June 1994.

15. M. Ujaldon, E. L. Zapata, B. M. Chapman, and H. P. Zima. New Data-Parallel Language Features for Sparse Matrix Computations. In Proc. of 9th IEEE International Parallel Processing Symposium, Santa Barbara, California, April 1995.

16. J. Wu, R. Das, J. Saltz, H. Berryman, and S. Hiranandani. Distributed Memory Compiler Design for Sparse Problems. IEEE Transactions on Computers, 44(6), 1995. 